\title{
Granule Characterization During Fluid Bed Drying by Development of a Near Infrared Method to Determine Water Content and Median Granule Size
}

\author{
Florentine J. S. Nieuwmeyer, ${ }^{1}$ Michiel Damen, ${ }^{1}$ Ad Gerich, ${ }^{1}$ Federica Rusmini, ${ }^{1}$ Kees van der Voort \\ Maarschalk, ${ }^{1,3}$ and Herman Vromans, ${ }^{1,2}$
}

Received December 19, 2006; accepted March 26, 2007; published online May 5, 2007

\begin{abstract}
Purpose. Water content and granule size are recognized as critical process and product quality parameters during drying. The purpose of this study was to enlighten the granule behavior during fluid bed drying by monitoring the major events i.e. changes in water content and granule size.

Methods. NIR spectra collected during drying and water content of sampled granules were correlated by principal component analysis (PCA) and partial least squares regression (PLSR). NIR spectra of dried granules were correlated to median granule size in a second PCA and PLSR.

Results. The NIR water model discriminates between various stages in fluid-bed drying. The water content can be continuously predicted with errors comparable to the reference method. The four PLS factors of the granule size model are related to primary particle size of lactose, median granule size exceeding primary particle size and amorphous content of granules. The small prediction errors enable size discrimination between fines and granules.

Conclusion. For product quality reasons, discrimination between drying stages and end-point monitoring is highly important. Together with the possibilities to determine median granule size and to distinguish fines this approach provides a tool to design an optimal drying process.
\end{abstract}

KEY WORDS: characterization; fluid bed drying; granule size; near infrared; water.

\section{INTRODUCTION}

Granulation is a common unit operation in manufacturing of oral dosage forms. Granules are often prepared by wet processes which have been extensively studied. Remarkably, the subsequent drying step has received relatively little attention. In batch production, drying is often the bottleneck either for time or product quality reasons. Plate drying is a slow process. In the fast fluid-bed drying process attrition, a size reduction phenomenon which leads to the formation of fines is considered a product quality diminishing side effect (1).

\footnotetext{
${ }^{1}$ Department of Pharmaceutics, NV Organon, P.O. Box 20, 5340 BH, Oss, The Netherlands.

${ }^{2}$ Department of Pharmaceutics, Utrecht Institute for Pharmaceutical Sciences (UIPS), Utrecht University, Utrecht, The Netherlands.

${ }^{3}$ Department of Pharmaceutical Technology and Biopharmacy, Groningen University Institute for Drug Exploration (GUIDE), Groningen, The Netherlands.

${ }^{4}$ To whom correspondence should be addressed. (e-mail: Florentine. nieuwmeyer@organon.com

ABBREVIATIONS: $\mathrm{d}_{10}$, cumulative $10 \%$ frequency undersize $[\mu \mathrm{m}]$ $\mathrm{d}_{50}$, cumulative $50 \%$ frequency undersize (median granule size) $[\mu \mathrm{m}]$; $\mathrm{d}_{90}$, cumulative $90 \%$ frequency undersize $[\mu \mathrm{m}]$; KF, Karl Fischer; NIR(S), near infrared (spectroscopy); PAT, process analytical technology; PC, principal component; PCA, principal component analysis; PLS, partial least squares; PLS(R), partial least squares (regression); SEC, standard error of calibration; SECV, standard error of cross validation; SEP, standard error of prediction.
}

Pharmaceutical granules exhibit an intrinsic breakage propensity during drying which is dependent on water content and extent of stress exposed to the granules (2). By use of an inline technique to monitor water content this critical process step can both be acknowledged and followed. Moreover granule characterization during fluid bed drying enables better process understanding which leads to better process control.

Process understanding receives more and more attention. This is because product quality assurance can only be achieved by thorough knowledge of the critical process steps. Therefore, process understanding and control using in-line analytical techniques such as near infrared spectroscopy (NIR) have received much attention (3-9). NIR spectra can contain information about both physical and chemical parameters which are both quantitatively and qualitatively interpretable (3-9). Furthermore NIR is fast, robust and non-invasive, requires no sample preparation and will not produce any waste products. Therefore NIR can be considered almost an ideal tool for process monitoring and control. Although NIR spectra might be fully spectroscopically explained, the subsequent chemometric modeling, interpretation and calibration of the data set in relation to the product or process parameters proves often a more difficult and time-consuming task, and does not provide a basic understanding of the process itself. It is however of significant importance to fully elucidate the interpretation of the chemometric fitting. As process understanding is a key goal of the Process Analytical Technology (PAT) initiative the control tools, such as NIR, should be fully 
understood as well. In other words; black box processes should not be monitored and controlled by black box methods.

NIR has often been applied for the determination of water content. In fluid-bed drying different phases are defined in relation to the evaporation rate of water: the constant rate period, the falling rate period and the equilibrium period (1). For process monitoring in fluid-bed drying the different phases in drying should be distinguished by the NIR model. For end point determination the known dry point should be acknowledged preferably with an error similar or smaller to the current used off-line water determination methods as Karl Fischer coulometry or heat balance methods. It is known that sodium tartrate with an absolute water content of $15.5 \%$ can be determined with a standard deviation of $0.3 \%$. Therefore a standard deviation of $0.3 \%$ is used as a comparison to the error obtained with the NIR method. Apart from following water content, NIR spectroscopy is theoretically able to follow other changes like granule size distribution $(4,8,10-12)$.

To distinguish granules from primary particles or fines in the NIR-granule size model the prediction error should be similar or smaller than the particle size of the original primary particles. Otherwise no distinction is possible between granules, primary particles or attrited material.

Water content and granule size are recognized as critical process and product quality parameters during drying processes. By NIR spectroscopy theoretically both water content and granule size can be monitored in-line. Granule characterization during the drying process will increase process understanding. The aim of this study was to develop a fully understood NIR model to monitor both water content and granule size during fluid-bed drying processes and thereby increase process understanding.

\section{MATERIALS AND METHODS}

\section{Drying Process}

\section{A. Fluid-bed Drying}

One point four kilograms Lactose 200M (DMV Veghel, The Netherlands) was granulated in a high shear mixer (Gral 10, Colette, Wommelgem, Belgium) for 10 min (mixer speed $450 \mathrm{rpm}$, chopper speed 1,500 rpm). Two hundred twentyfive milliliters de-mineralized water was used as binding liquid. The water was poured onto the moving powder bed. The wet granules were transferred to a fluid-bed dryer (Granulator Niro Aeromatic MP-1, Gea, Bubendorf, Switzerland). To obtain a stable temperature during the drying process, the equipment was heated up for $30 \mathrm{~min}$ before use with the following parameters: inlet temperature $60^{\circ} \mathrm{C}$, air flow rate $60 \mathrm{~m}^{3} / \mathrm{h}$. In the fluid-bed drying experiments inlet air temperature $\left(40,50\right.$ or $\left.60^{\circ} \mathrm{C}\right)$ and inlet air flow $(60,70,80$ or $90 \mathrm{~m}^{3} / \mathrm{h}$ ) were varied. All the different combinations of parameters were explored in order to evaluate the influence on drying rate and subsequent water content. The drying process was extended for $20 \mathrm{~min}$. Inlet and outlet temperature and relative humidity were monitored throughout every experiment. The experiments were monitored in-line with a NIR probe as described below.

\section{B. Microwave Drying}

Eleven kilograms Lactose 200M (DMV Veghel, The Netherlands) was granulated in a high shear mixer (Gral 75 / Vactron, Colette, Wommelgem, Belgium) for 5 min (mixer speed $130 \mathrm{rpm}$, chopper speed 1,500 rpm) with $1,700 \mathrm{ml}$ of water as binding liquid. Vacuum (50 $\mathrm{kPa}$, absolute value) was applied and the granules were exposed to microwave radiation $(3 \mathrm{~kW}$, electric field $100 \%)$ until the granules were dry. This point is characterized by a steep increase in back reflection and decrease in applied power to a steady state situation. Microwave drying can currently not be monitored in-line by NIR spectroscopy. Spectra of dried samples are collected off-line. The microwave dried samples were used as reference.

\section{Data Acquisition and Pre-treatment Water Measurement}

The NIR probe (FOSS NIRSystem Analyzer, software package Vision ${ }^{\circledR}$, U.K.) was placed into the wall of the fluidbed dryer at the same height of the system integrated sampling probe. Probe position was optimized by spectral analysis to ensure continuous, reliable and accurate monitoring. The NIR probe was secured tightly in the wall of the vessel to obtain stable recordings and to prevent influence of the probe on the process or the product. In-line diffuse reflectance NIR spectra were recorded for each fluid-bed drying experiment. The drying process was continuously monitored by means of NIR recordings every $30 \mathrm{~s}$, starting at the same time point as the drying process. Each spectrum is the average of 32 individual scans in the wavelength region between 1100 and $1900 \mathrm{~nm}$, with a resolution of $2 \mathrm{~nm}$. NIR data analysis was done using Unscrambler@, version 9.2 (Camo ASA, Oslo, Norway). Starting at $t=0$, every minute a sample for reference water content determination was collected. The water content of the granules was determined with an automated Karl Fischer titration (Metrohm KF coulometer $756 \mathrm{~K}$ with oven sample processor 774 working at $150^{\circ} \mathrm{C}$, Herisau, Switzerland). The coulometer has been calibrated before every analysis (maximum of 28 samples) with sodium ditartrate (mean water content $15.5 \%$, sd $0.3 \%$ ) To determine the optimum wavelength interval or precise wavelength to correlate spectra data to water content the influence of water on NIR spectra was considered. Water or hydrogen has a large absorption band around 1,420 nm $(6,7)$. The water band basically consists of multiple overlapping bands. The exact position of these bands is dependent on the nature of the samples. The shift of the overtone bands is related to the changes of the individual bands forming the combined band (13). Static NIR spectra of each collected sample were recorded and compared to the recorded in-line spectra. In this way the correlation between each NIR spectrum and the corresponding water content value was confirmed. By comparison of principal component analysis (PCA) and partial least square regression (PLSR) based upon in-line or off-line spectra it was confirmed that by the probe position and load of the bed similar spectra were recorded for in-line and off-line spectra. In total, 163 reference samples and spectra were used. For the water content correlation the 163 spectra were normalized (SNV) and transformed to first derivative spectra by means of Savitzky-Golay algorithm prior to PLS regression analysis. 
Data Acquisition and Pre-treatment NIR-median Granule Size Model

For the granule size model fully dried granules were used. The granules were sieved in size fractions (Retsch control 200 ' $\mathrm{g}$ ', sieve, Haan, Germany). Granule sizes $\left(d_{10}, d_{50}, d_{90}\right)$ were determined by laser diffraction in threefold (Malvern Mastersizer S. (U.K.)), using a 1,000 $\mathrm{mm}$ lens with 2-propanol as dispensing medium (Fraunhofer analysis). To determine the primary particle composition of the granules, agglomerates were dispersed in 2-propanol and placed on an ultrasonic bath for a total of $2 \mathrm{~h}$. After $2 \mathrm{~h}$ the distribution of the de-agglomerated granules did not change anymore. It was shown that the ultrasonic treatment did not influence the primary particle size of lactose $200 \mathrm{M}$ by measuring the particle size distribution before and after the full de-agglomeration time. Off-line spectra of the sieve fractions of fluid-bed dried and microwave dried granules were collected. To obtain optimal spectral information a Nicolet Antaris FT-NIR RTS was used (Thermo Electron, Madison, USA), wavelength area $1000-2500 \mathrm{~nm}$, resolution $1.6 \mathrm{~nm}$, number of scans per spectrum, 32). To enable a comparison with the NIR probe the used wavelength area for analysis and modeling was 1100-1900 nm. Four independent batches of fluid-bed dried granules were used. One batch of microwave dried granules was used as reference batch. This reference was used to detect possible influence of the drying process on the model not detected with the four batches containing fluid-bed dried granules, i.e. to assess the robustness of the model. For the granule size model the 64 derived spectra were not preprocessed prior to PCA and PLS regression analysis. 64 full zero order data spectra (42 fluid-bed dried and 22 microwave dried samples) obtained from fully dried and sieved granules were used for calibration and validation of the PLS model. The influence of fines and attrition was tested by adding extra fines to the granule samples. The influence of amorphous content of the granules was tested by NIR measurement of fully crystalline and fully amorphous fractions. One hundred percent amorphous freeze dried lactose was generously provided by DMV-Frontera, Veghel, The Netherlands.

\section{Strategy Multivariate Data Analysis and Modelling}

PCA analysis was used to interpret the obtained spectra in relation to the reference value qualitatively. The relevance of the data points and the presence of possible outliers were analyzed by comparison of leverage and residual variance (influence plot). If outliers solely based upon the spectral data were detected, they were more thoroughly investigated and if necessary removed.

Calibration of the PLS model was quantified by standard error of calibration (SEC), indicating the fit between NIR spectra and off-line values. Validation was done by full crossvalidation. The NIR-median granule size model was validated and tested with seven independently granulated and fluid-bed dried granule samples. During validation, the standard error of cross validation (SECV) was calculated. It is expected that the SECV represents the SEP (standard error of prediction). The SEP is used to evaluate the uncertainty of the future predictions. To avoid influence from outliers on the PLSR based upon the reference method (Karl Fischer or laser diffraction), all reference values and methods were checked, and if necessary removed from the data set.

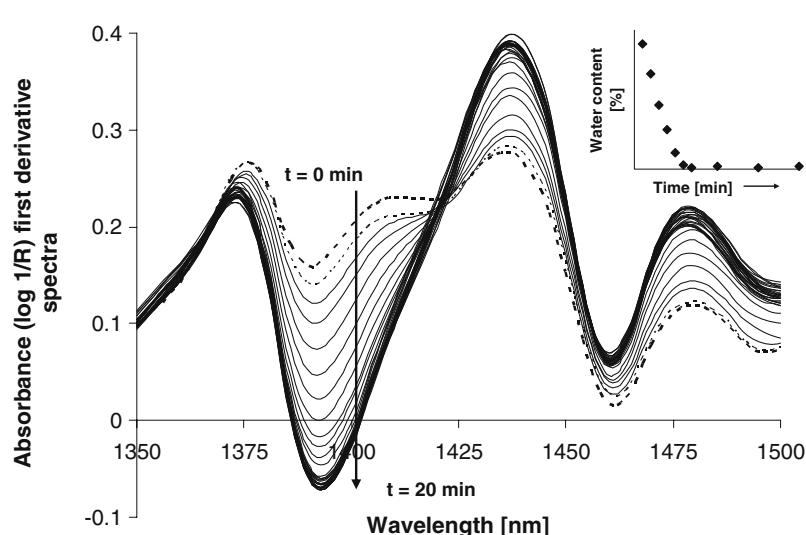

Fig. 1. In-line NIR first derivative spectra of lactose granules during one fluid-bed drying experiment inlet air temperature $60^{\circ} \mathrm{C}$, air flow $60 \mathrm{~m}^{3} / \mathrm{h}$. One NIR spectrum is an average of 32 scans per $30 \mathrm{~s}, t=0$ thick dashed line spectrum, highest water content, $t=30 \mathrm{~s}$ thin dashed line spectrum, $t=1 \mathrm{~min}$ very thin dashed line spectrum etc. In the small inserted graph the change in absolute water content of the sampled granules as determined by Karl Fischer analysis in time is shown.

\section{RESULTS AND DISCUSSION}

\section{NIR-water Content Model}

In Fig. 1 the spectra of the drying granules during one fluid-bed drying experiment are shown. The spectra were collected every $30 \mathrm{~s}$ and therefore represent the fluid bed drying process in time starting with wet granules (thick dashed line in Fig. 1) to fully dry granules (overlying spectra). During drying granules were sampled every minute. The water content of sampled granules was determined. The results were correlated to the obtained NIR spectra. The peaks observed in this wavelength interval are known to correlate to water, either free or bound $(6,7,13)$. In Fig. 1 the distance between the curves around $1,400 \mathrm{~nm}$ (first overtone $\mathrm{O}-\mathrm{H}$ in $\mathrm{H}_{2} \mathrm{O}$ ) from top to bottom first is constant, then declining and finally zero. As the water content of the sampled granules and the time interval between collection of the spectra are known, (Fig. 1), the distances between the spectra seem to be correlated to a change in water content. A constant, declining and zero distance variation is then related to respectively the constant rate, falling rate and equilibrium period during the fluid-bed drying process.

PCA revealed that all spectral data were primarily correlated to one principal component (data not shown). The analysis revealed no more distinctive features of the data. Thereupon the data set was used in the PLS regression.

The 1-factor PLS fit from data of the wavelength interval 1350-1500 $\mathrm{nm}$ appeared to be superior to that using the single $1400 \mathrm{~nm}$ data only. It gives the lowest standard error of calibration (SEC) and standard error of cross validation (SECV) with the highest correlation. Fig. 2a shows the fit between the determined (Karl Fischer) and predicted (NIR) water content. The large number of dry granule samples obtained during drying under various process conditions (21 experiments, inlet air temperature $40-60^{\circ} \mathrm{C}$ and air flow $60-90 \mathrm{~m}^{3} / \mathrm{h}$ ) accounts for the large data point cloud at low water content (left in Fig. 2a). In all experiments granules were all completely dried after $10 \mathrm{~min}$. All the drying processes were extended to $20 \mathrm{~min}$. NIR spectra were recorded every $30 \mathrm{~s}$ and therefore many data points for dry granules were obtained. The SECV for the dried 


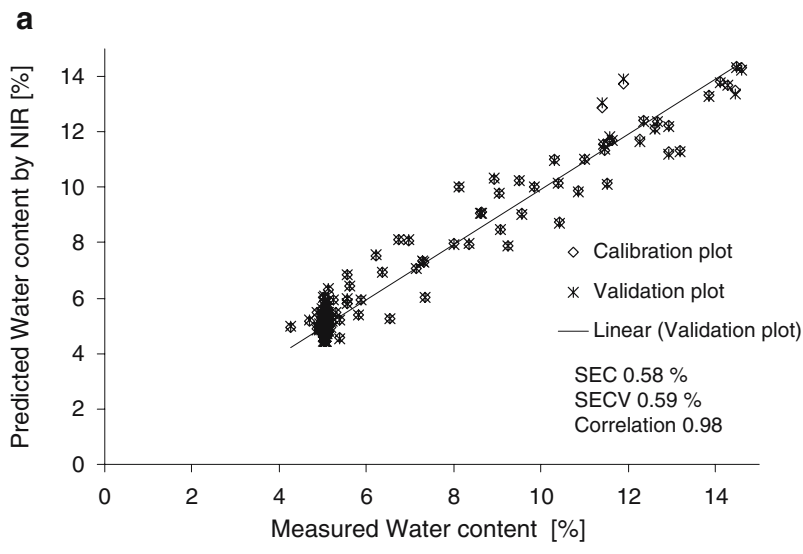

b

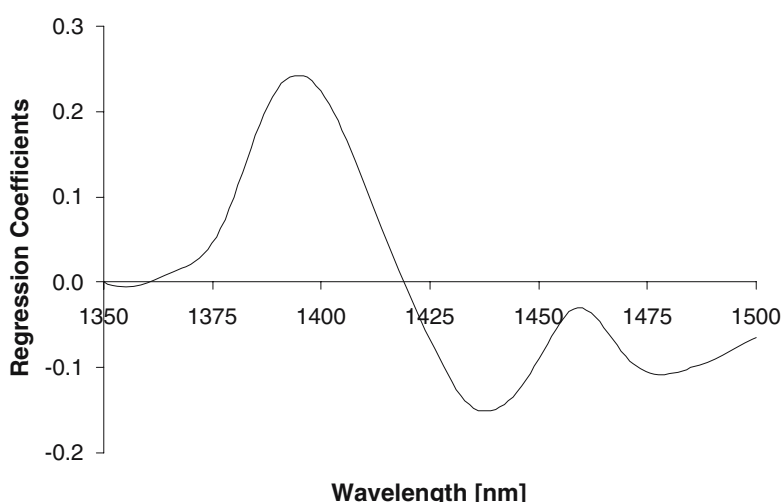

Fig. 2. a PLS regression of measured water content reference values (Karl Fischer) and NIR predicted water content of lactose granules in the fluid-bed drying process from the total data set (21 fluid-bed drying experiments, inlet air temperature $40-60^{\circ} \mathrm{C}$, air flow $60-90 \mathrm{~m}^{3} / \mathrm{h}$, no influence of process conditions are observed). b NIR-PLS regression coefficients plot of the model.

granules (water content up to $5.2 \%$ ) is $0.28 \%$. This small error is beneficial for an accurate in-line end-point determination of the drying processes.

The regression coefficients plot (Fig. 2b) represents the maximum spectral response to change in granule water content. Logically, the maximum spectral variation is the difference between the spectra of the wettest (the thick discontinuous line) and the driest granules (the overlying spectra) in Fig. 1.

As can be seen only one PLS factor is needed to yield an acceptable fit between spectral data and water content. If more factors would be needed to describe a single change this would suggest the existence of a more complex correlation where solely decreasing water content is not the only determinant of the observed changes in the spectra (Error! Bookmark not defined.). The inclusion of more than one PLS factor did not improve the correlation, but did increase the inclusion of noise. In the obtained model, $85 \%$ of the spectral variance is correlated with $96 \%$ of the water variance. From the score plot (not shown) and the regression coefficients plot it is concluded that the various process conditions as air flow rate and inlet air temperature do not affect the prediction of water content by NIR in any way. The data points per experiment are all evenly distributed throughout the plots. The SEC and SECV of the NIR water content model are comparable to the reported relative standard deviation of the reference method (Karl Fischer) (14). For

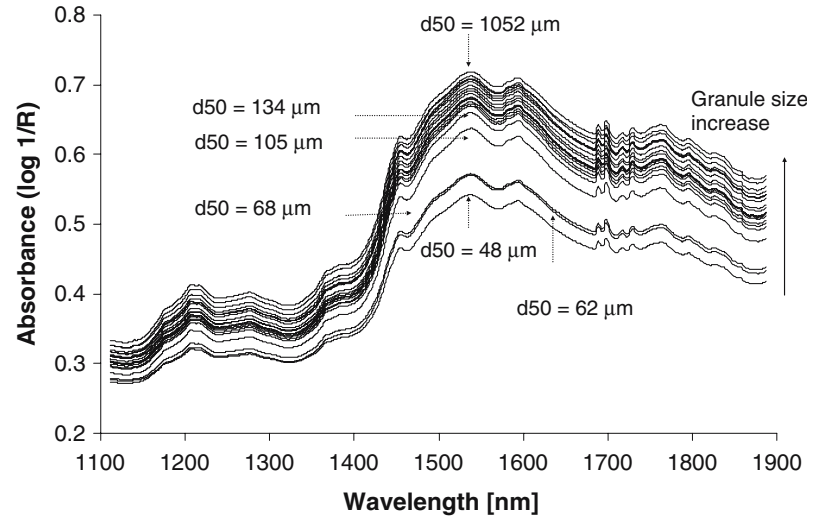

Fig. 3. Full data 0 -order spectra of lactose fluid bed dried granules with size range $48-1,052 \mu \mathrm{m}\left(\mathrm{d}_{50}\right)$. For clarity reasons not all available spectra of the data set are shown, but only the spectra collected of granules of one batch. The smallest granule size corresponds to the lowest spectrum in the figure. Absorbance increases upon granule size increase.

dryer granules the SECV is even smaller than the reported value. Therefore the model is considered a valid in-line alternative to the off-line Karl Fischer water content determination.
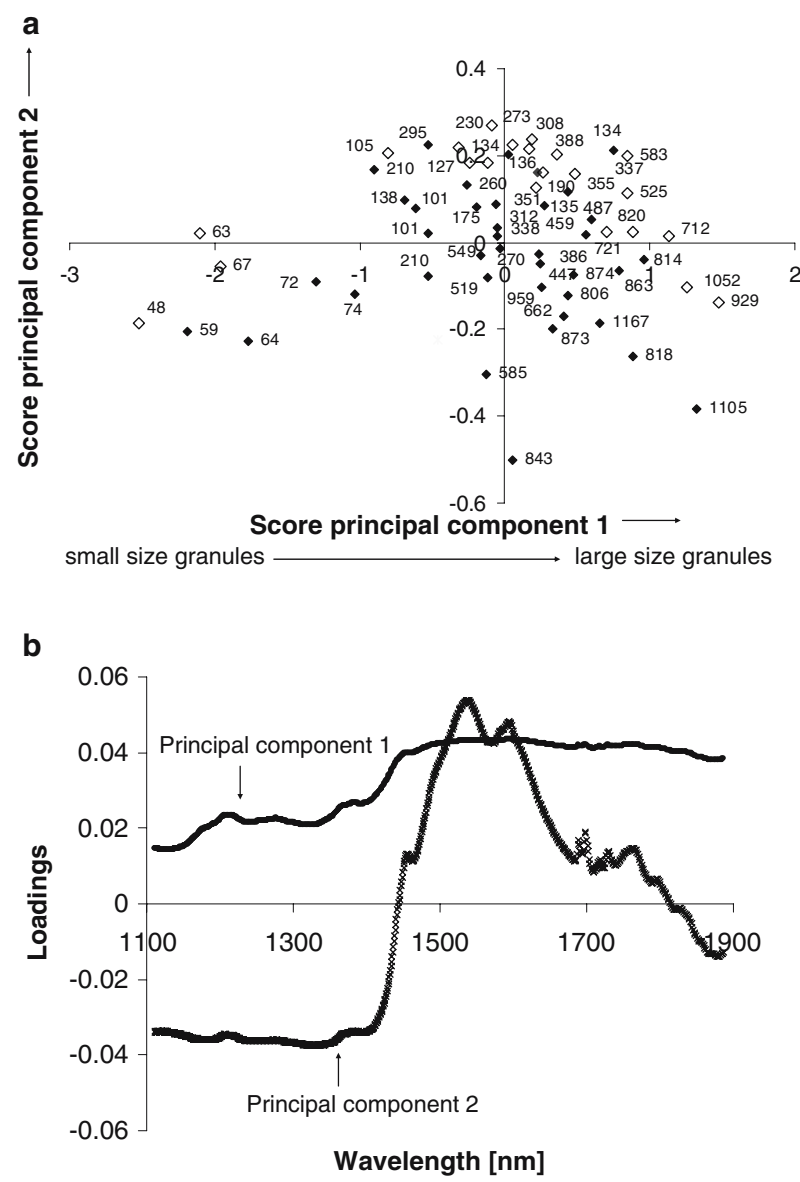

Fig. 4. a Plot of PCA NIR spectra lactose granules with size range 48-1,052 $\mu \mathrm{m}$. The closed squares correspond with spectra from fluidbed dried granules, open squares represent microwave vacuum dried granules. b x-loadings plot of the two principal components. 


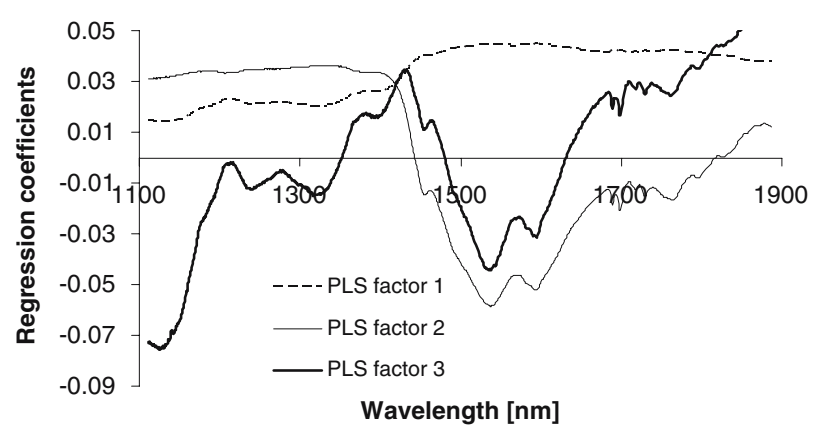

Fig. 5. Regression coefficients plot of three of the four PLS factors used to correlate NIR data to granule size (For clarity only three PLS factors are shown).

\section{NIR-median Granule Size Model}

\section{Principal Component Analysis}

For end point determination of fluid-bed drying processes not only the water content of the granules but also median granule size and the formation of fines are important. With NIR it is possible to measure both water content and granule size simultaneously by use of different models. The direct effect of increasing granule size (dry granules) upon spectral absorption can be observed in Fig. 3. Upon increasing granule size diffuse reflectance decreases and consequently increased absorbance is observed. Each spectrum corresponds to a different size class wherein the median granule size varies between 48 and $1,052 \mu \mathrm{m}$. The difference between the spectra of the smallest and the largest granules is remarkable; large differences in absorbance level are observed between the granules of the smallest size classes, whereas this difference decreases upon size enlargement. To specify the influence of the increasing granule size on the NIR spectra further PCA is performed.

The principal component analysis of the 64 spectra reveals that two principal components (PCs) sufficiently describe variation of the spectra (Fig. $4 a$ and b). In the score plot (Fig. 4a) the first principal component (horizontal axis) covers the whole spectral data set correlated to granule size from the smallest to the largest particles (left to right). Data points located close to the horizontal axis are better described by the first PC than particles located further away from the axis. Ninety-six percent of the spectral variation is described by the first PC, whereas the second PC describes only $4 \%$ variation. The first PC describes the most important variation in the total data set, but the remaining $4 \%$ is enough to investigate the nature of the second PC more thoroughly. The second PC (vertical axis, from top to bottom) concerns data points correlated to granules with somewhat larger median granule size (i.e. 134-1,167 $\mu \mathrm{m}$ ). As for the first PC; data points located further from the vertical axis are less well described by the second PC. This implies that the data points correlated to the smallest granule size are less well described by the second PC.

The x-loadings plot (Fig. 4b) shows that the first PC has a positive value throughout the whole plot at every wavelength, which indicates a constant offset variation. This gives a strong correlation with the continuously increasing granule size. The second PC has very distinctive peaks around 1540, 1600, 1700,

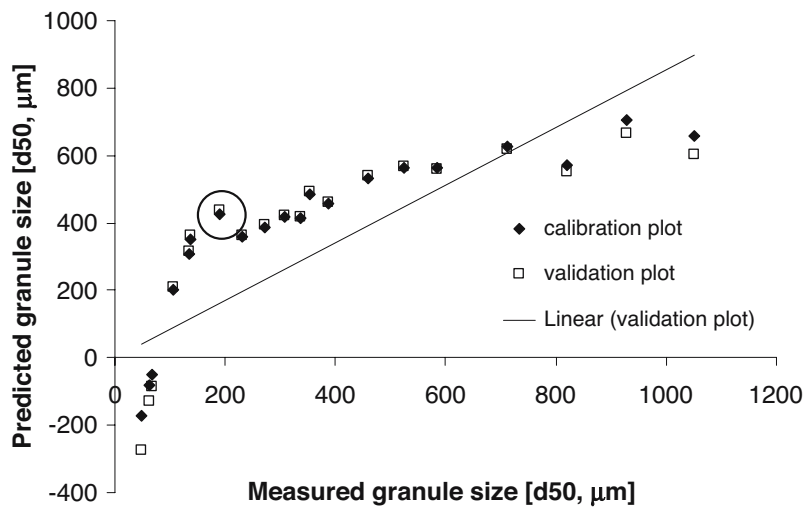

Fig. 6. Correlation between NIR and granule size based upon the first PLS factor seen in Fig. 5.

1720 and $1730 \mathrm{~nm}$. The chemical content of the granules does not change throughout the fluid bed drying experiments. Therefore the sharp peaks can only be related to physical changes in the granules. This will be elaborated hereafter.

\section{Partial Least Squares Regression}

To find a potential fit between the spectral information and the median granule size PLSR was performed. When the individual NIR spectra of fluid bed dried sieved granules are correlated to the median granule size of the granules by PLSR, an appropriate fit can be derived $\left(R^{2}=0.98\right.$, $\mathrm{SECV}=43.0 \mu \mathrm{m}, \mathrm{SEC}=34.0 \mu \mathrm{m})$. The NIR spectra of the microwave dried sieved granule fractions with a narrow size margin yield a similar model $\left(R^{2}=0.99, \mathrm{SECV}=46.2 \mu \mathrm{m}\right.$, $\mathrm{SEC}=36.3 \mu \mathrm{m})$. The score plots and regression coefficients plot were comparable. The microwave dried granules are used to detect possible influences of the drying technique upon the spectral information. Differences in granule characteristics, based upon drying method, can potentially have a large influence on the NIR scattering and absorbance. These influences are not detected by the current method. Therefore the two different size sample sets were combined in one NIR granule size fit (64 spectra with corresponding median granule size determined by laser diffraction). The calibration- and prediction error for this model are each small $\left(R^{2}=0.98, \mathrm{SECV}=70.4, \mathrm{SEC}=59.6\right)$, In the regression

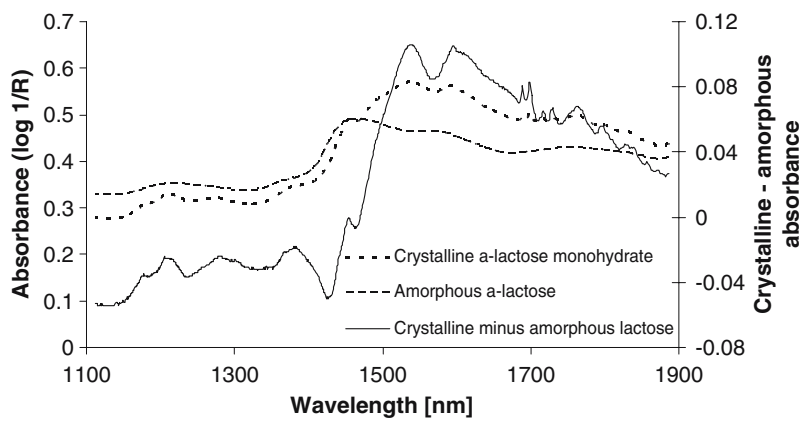

Fig. 7. Spectra of $100 \%$ crystalline (lactose $200 \mathrm{M}$ ) and $100 \%$ amorphous (freeze dried) lactose. The spectrum of the amorphous lactose is characterized by the presence of broad peaks and absence of sharp peaks. 
Table I. Change in Primary Particle Composition of Different Sized Microwave Dried Lactose Granules

Size

\begin{tabular}{|c|c|c|c|c|c|c|c|}
\hline Original granule size $\left(d_{50}\right)[\mu \mathrm{m}]$ & 48.1 & 57.4 & 66.8 & 230.0 & 337.0 & 458.7 & 1052.0 \\
\hline Primary Particle size $\left(\mathrm{d}_{50}\right)[\mu \mathrm{m}]$ & 44.2 & 33.4 & 51.7 & 20.4 & 23.0 & 5.8 & 7.6 \\
\hline
\end{tabular}

coefficient plot (Fig. 5) the different factors are shown, required to correlate the NIR spectra with the granule size. From Fig. 5 it can be concluded that PLS factor 1 and 2 show a high resemblance with PC 1 and 2 as seen in Fig. 4b. The optimum fit between median granule size and the spectral data is gained taking four PLS factors. For clarity reasons only three PLS factors are shown in Fig. 5. As with the water model one variable (granule size) is considered versus the spectral data. Therefore it is interesting that so many factors are needed to achieve an optimum correlation. The first PLS factor describes $95 \%$ of the total variance in $\mathrm{x}$ values (NIR) but only $55 \%$ of granule size effects variance. The second component designates $4 \%$ of $\mathrm{x}$-variance and $24 \%$ of granule size variance. The third and fourth components represent only a very small part of the spectral variance but each cover $7 \%$ size variance. When so many factors are needed for an adequate correlation further revelation of the characteristics of the PLS factors is necessary.

A fit based on the first principal component only has some very distinctive features as seen in Fig. 6; granules with a granule size larger then $136 \mu \mathrm{m}$ (encircled), all have a fairly constant predicted granule size around $400 \mu \mathrm{m}$ according to the fit. Lactose $200 \mathrm{M}\left(\mathrm{d}_{10} 9.6 \mu \mathrm{m}, \mathrm{d}_{50} 40.1 \mu \mathrm{m}, \mathrm{d}_{90} 104.4 \mu \mathrm{m}\right)$ has a wide size distribution with the largest particles $\left(d_{90}\right)$ just above $104 \mu \mathrm{m}$. It is therefore evident to relate the first PLS factor to the size of the primary particles and the smaller granules. Furthermore the first PLS factor has a constant positive value and is therefore related to the constantly increasing granule size as seen in Fig. 5. Figure 3 exhibits large differences between the raw spectra for small granules, whereas for larger granules small differences are observed. This suggests that large granules and small granules are described by different factors. This is the reason that more than one PLS factor is needed to obtain an appropriate correlation.

In Fig. 4a the PC data are positioned to elucidate the distinction between the individual spectra. From Fig. 4a it is seen that the data points relate to the $\mathrm{d}_{50}$, although the first PC exhibits a more obvious correlation than the second PC. In order to obtain an idea of the nature of the second PC a closer look at the spectra may yield some indications: The second and third PLS factor have very distinctive peaks around 1540, 1575, 1597, 1686, 1696, 1715, 1725, 1745, 1794 and 1827 nm (Fig. 5). From 1540 to $1900 \mathrm{~nm}$ these two PLS factors are the mirrored image of PC 2. The sharp peaks in the spectra can be correlated to the orientation of an intact crystal lattice. Upon increasing crystal lattice disorientation often less sharp or flattened peaks are observed (15). It is hypothesized that granules can have variations in crystal orientation related to granule size. It is known that upon solidification of lactose solutions (as in spray drying) amorphous lactose can be formed $(16,17)$. To investigate the possibility of the existence of amorphous lactose in the granules, the zero order NIR spectra of $100 \%$ amorphous lactose (freeze dried lactose) and $100 \%$ crystalline lactose were collected (Fig. 7). NIR has often been used for quantitative determination of the crystallinity of lactose $(15,18-20)$.
If PLS factor 2 and 3 are susceptible to physical features of the granules and if it is assumed that increasing amorphous content is included, the PLS factors should be related to the difference between the spectra of $100 \%$ crystalline and $100 \%$ amorphous lactose. When the spectrum of the amorphous lactose is subtracted from the spectrum of the crystalline lactose a "spectrum" remains with great similarities to PLS factor 2 and 3. This similarity is seen in the 1690-1770 nm region characterized by sharp bands. Especially PLS factor 3 has more similarities with the resulting "spectrum" over the whole wavelength range with corresponding peaks around 1146, 1175, 1206, 1240, 1277, 1332 and $1380 \mathrm{~nm}$. The similarities of the PLS factors 2 and 3 with the subtraction spectrum seems to argue that a direct relation to the amorphous content of the granules exists. From the above this would mean that the amorphous lactose content could increase upon increasing granule size. This hypothesis is further tested by further granule characterization. As shown by Van den Dries et al. (21) and Scott et al. (22) large granules can be preferentially built up from fine primary particles and small granules from large primary particles. The primary particle composition of the lactose granules was determined by ultra sound dispersion of the granules and subsequent laser diffraction size measurements. The difference in composition is shown in Table I. Large granules with a median size of $1,052 \mu \mathrm{m}$ consist of primary particles with a median granule size of $7.6 \mu \mathrm{m}$. Very small granules with a median size of $48.1 \mu \mathrm{m}$ consist of primary particles with a median size of $44.2 \mu \mathrm{m}$. These measurements show that the largest granules are indeed composed of small primary particles whereas small granules consist of large primary particles. If granules are composed of relatively small particles a larger internal surface will be expected as a consequence of which the granule contains relatively more water. Therefore wet small granules do contain relatively less water than larger wet granules. This was confirmed by determination of the water

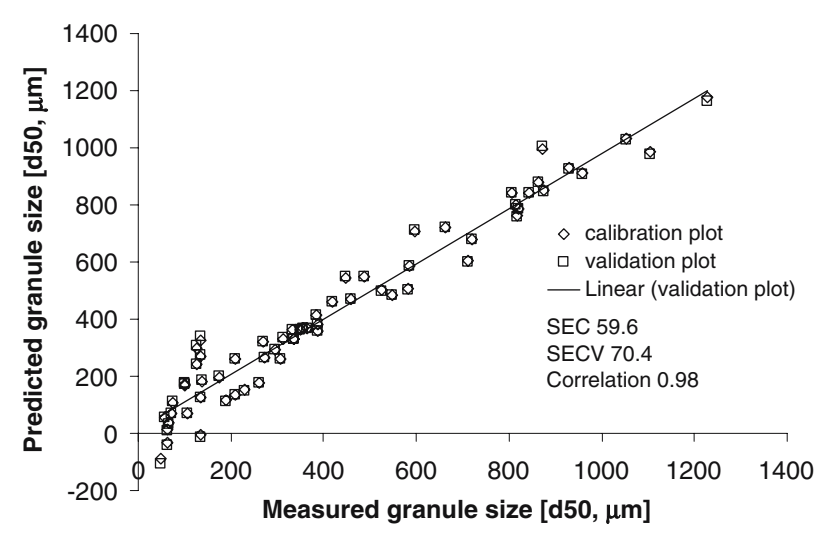

Fig. 8. PLS regression fit correlating measured median granule size (laser diffraction method) to predicted median granule size (NIR). 
content of wet granules of four separate high shear granulated batches. Wet granules were sieved in a closed system to prevent evaporation. The water content of the sized granules was determined by Karl Fischer. Every decreasing granule size class of sieved wet granules appeared to have a decreasing water content (student's paired $t$-test, $n=4, p \leq 0.05$, granule sieve classes 2,500-3,350 $\mu \mathrm{m}, 1,250-2,500 \mu \mathrm{m}, 850-1,250 \mu \mathrm{m}$, 600-850 $\mu \mathrm{m}$ and $450-600 \mu \mathrm{m})$.

Upon drying of saturated lactose solutions the formation of amorphous lactose has been shown by Farber et al. (16). Therefore higher relative amorphous lactose content, formed upon drying of the lactose solution is possible. From the above it is concluded that upon increasing granule size more amorphous lactose can be present. Small granules consisting of large crystalline primary particles exhibit a minor internal surface and are therefore almost fully crystalline. As stated earlier, PC 2 describes larger granules exceeding primary particle size. Therefore primary particles are less well described by PC 2 and corresponding PLS factor 2 and 3.

Although with the current method the degree of crystallinity is based upon the chemometric interpolation between fully crystalline and fully amorphous lactose, the exact character of the amorphous content is not revealed. In a two phase model a mixture of amorphous and crystalline regions is assumed whereas in a one phase model a continuous transition with increasing lattice disorder from a fully crystalline to a $0 \%$ crystalline state is expected $(23,24)$. If it is believed that the primary particles do not physically change, the only location of the amorphous content will be between these primary particles. As the transition to crystalline is a time-consuming process up to several days or even weeks (16) the chance that a physical mixture of fully crystalline and fully amorphous regions is present is small. Regions with disordered lattice structures are more likely to occur.

Next to an understanding of the nature of the relation between PLS factors and characteristics of the analyzed substance a good PLS model should also meet requirements towards the calibration error, prediction error and correlation. To distinguish fines from granular material the SEC and SECV should be small. If the calibration and prediction error are large this distinction worsens. In Fig. 8 the finally derived model is shown. Small particles are predicted less well than the larger granules. This is confirmed by the use of independently obtained validation samples consisting of granules spanning the full calibration range of particle sizes. Granule samples with a median granule size between 300 and $800 \mu \mathrm{m}$ are predicted best. Samples containing only granules and primary particles smaller than $53 \mu \mathrm{m}$ are predicted negatively according to the model, but with a SECV of 128 and a correlation of still 0.96 . The attractive consequence of this is that attrition phenomena, characterized by excessive formation of fines, will be recognized easily. For in-line monitoring of the fluid-bed drying process this is a desired feature as attrition is unwanted for product quality reasons.

\section{Combined Applicability of Models}

Currently the correlation between NIR and water content can be used to do process monitoring and end-point determination. For granule size determination humidity of the granules has been proven substantially influential to the
NIR spectrum (11). Furthermore it is shown above that larger granules contain more water than smaller granules. A more extensive data set has to be developed to be able to make the correlation between NIR and the granule size of wet granules. Under most process circumstances granules are dried after $10 \mathrm{~min}$ to such an extent that interference of water on the full spectrum is minimal. This point can easily be detected by NIR monitoring of the granule water content during the process. The NIR method to monitor the granule size and attrition effects can then be used when the influence of water is less pronounced.

\section{CONCLUSION}

For product quality reasons, discrimination between drying stages and end-point monitoring is highly important. The currently developed NIR granule water content model provides an effective real time method for monitoring of the fluid bed drying process. The low SEC and SECV and high correlation coefficient confirm the reliability of the fit. The correlation is independent of process conditions. Although it is demonstrated that differences in water content exist as a function of granule size, the estimated water content represents a median value of the water content of the granules. NIR monitoring can replace currently used off-line techniques. The relative error of the NIR determination is comparable to Karl Fischer analysis. The end-point of the drying process can be adequately acknowledged. The endpoint can be determined faster than with current timeconsuming off-line techniques. The NIR granule size model is based upon four PLS factors. The first factor is related to the primary particle size and small granules. The second factor is related to the larger granules and the amorphous content of the granules. With these two parameters $100 \%$ of the spectral information and $79 \%$ of the granule size variation is included by the model. Information related to the granule size is revealed by factor 3 and 4 which both correlate another $7 \%$ of the granule size information to the model. The character of these two factors is also related to the amorphous content in the granules. The fit provides an effective method to monitor granule size and attrition effects. This approach provides a tool to design an optimal drying process.

\section{REFERENCES}

1. D. N. Travers. Drying. In M. E. Aulton (ed.), PharmaceuticsThe Science of Dosage Form Design, Churchill Livingstone, Edinburgh, 1988, pp. 629-646.

2. F. J. S. Nieuwmeyer, H. Vromans H. Granule breakage during drying processes. Int. J. Pharm. 329:81-87 (2007).

3. R. L. Green, G. Thurau, N. C. Pixley, A. Mateos, R. A. Reed, and J. P. Higgins. In-line monitoring of moisture content in fluid bed dryers using NIR spectroscopy with consideration of sampling effects on method accuracy. Anal. Chem. 77:45154522 (2005)

4. W. P. Findlay, G. R. Peck, and K. R. Morris. Determination of fluidized bed granulation end point using near-infrared spectroscopy and phenomenological analysis. J. Pharm. Sci. 94:604-612 (2004).

5. J. P. Higgins, S. M. Arrivo, and R. A. Reed. Approach to the determination of hydrate form conversions of drug compounds and solid dosage forms by near-infrared spectroscopy. J. Pharm. Sci. 92:2303-2316 (2003).

6. G. X. Zhou, Z. Ge, J. Dorwart, B. Izzo, J. Kukura, G. Bicker, and J. Wyvratt. Determination and differentiation of surface and 
bound water in drug substances by near infrared spectroscopy. $J$. Pharm. Sci. 92:1058-1065 (2003).

7. J. Beyer, and K. Steffens. Kalibrationsmodelle zur Bestimmung des Wassergehaltes pharmazeutischer Hilfsstoffe mittels NahInfrarot-Spektroskopie (NIRS). Pharm. Ind. 65:186-192 (2003).

8. P. Frake, I. Gill, C. N. Lusacombe, D. R Rudd, J. Waterhouse, and U. A. Jayasooriya. Near-infrared mass median particle size determination of lactose monohydrate, evaluating several chemometric approaches. Analyst 123:2043-2046 (1998).

9. J. Rantanen, O. Antikainen, J. Mannermaa, and J. Yliruusi. Use of near-infrared reflectance method for measurement of moisture content during granulation. Pharm. Dev. Techn. 5:209-217 (2000).

10. A. Gupta, G. E. Peck, R. W. Miller, and K.R. Morris. Nondestructive measurements of the compact strength and the particle size distribution after milling of roller compacted powders by near-infrared spectroscopy. J. Pharm. Sci. 93:1047-1053 (2004).

11. J. Rantanen, H. Wilkström, R. Turner, and L. S. Turner. Use of in-line near-infrared spectroscopy in combination with chemometrics for improved understanding of pharmaceutical processes. Anal. Chem. 77:556-563 (2005).

12. M. C. Pasikatan, J. L. Steele, C. K. Spillman, and E. Haque. Near infrared reflectance spectroscopy for online particle size analysis of powders and ground materials. Near Infrared Spectrosc. 9:153-164 (2001).

13. J. S. Schenk, J. J. Workman Jr., and M. O. Westerhaus. Application of NIR spectroscopy to agricultural products. In D. A. Burns, and E. W. Ciurczak (eds), Handbook of Nearinfrared Analysis Second edition, revised and expanded, Marcel Dekker, New York, 2001, pp. 383-431.

14. M. von Kloeden., K. Fischer. Water determination in solids, with the 774 oven sample processor using the 756 coulometer. Methrohm Ltd. CH-9101 Herisau, 1999.
15. L. NØrgaard, M. T. Hahn, L. B. Knudsen, I. A. Farhat, and S. B. Engelsen. Multivariate near-infrared and Raman spectroscopic quantification of the crystallinity of lactose in whey permeate powder. Int. Dairy. J. 15:1261-1270 (2005).

16. L. Farber, G. I. Tardos, and J. N. Michaels. Evolution and structure of drying material bridges of pharmaceutical excip ients: studies on a microscope slide. Chem. Eng. Sci. 58:45154525 (2003).

17. O. C. Chidavaenzi, G. Buckton, and F. Koosha. The effect of cospray drying with polyethylene glycol 4000 on the crystallinity and physical form of lactose. Int. J. Pharm. 216:43-49 (2001).

18. G. Buckton, E. Yonemochi, J. Hammond, and A. Moffat. The use of near infra-red spectroscopy to detect changes in the form of amorphous and crystalline lactose. Int. J. Pharm. 168:231-241 (1998).

19. R. A. Lane, and G. Buckton. The novel combination of dynamic vapour sorption, gravimetric analysis and near infra-red spectroscopy as a hyphenated technique. Int. J. Pharm. 207:49-56 (2000).

20. S. E, Hogan, and G. Buckton. The application of near infrared spectroscopy and dynamic vapor sorption to quantify low amorphous contents of crystalline lactose. Pharm. Res. 18:112116 (2001).

21. Dries. K. van den. The Paradox of high shear granulation; the formation of non-homogeneous granules. PhD thesis. 2004. ISBN 90-393-3654-7.

22. A. C. Scott, M. J. Hounslow, and T. Instone. Direct evidence of heterogeneity during high shear granulation. Powder Technol. 113:205-213 (2000).

23. R. Hüttenrauch. Molekulargalenik als grundlage moderner arzneiformung. Acta Pharm. Techn. Suppl. 6:55-127. (1978).

24. R. Suryanarayanan, and G. Mitchell. Evaluation of two concepts of crystallinity using calcium gluceptate as a model compound. Int. J. Pharm. 24:1-17 (1985) 